\title{
Contingency Detection in Multi-Agent Interactions
}

\author{
James Staley \\ Tufts University \\ James.Staley625703@tufts.edu
}

\begin{abstract}
Contingency is the principle of interaction that governs how one partner influences another. This paper presents a formalization for multi-agent contingency detection over discrete intervals of humanagent interaction. A multi-agent virtual sandbox is implemented for a human participant to play with one of two agents and produced labelled interaction data. We perform a preliminary analysis to detect contingency from continuous behavioral signals from the participant and agents.
\end{abstract}

\section{KEYWORDS}

human-robot interaction; contingency detection

\section{ACM Reference Format:}

James Staley and Elaine Schaertl Short. 2021. Contingency Detection in Multi-Agent Interactions. In Companion of the 2021 ACM/IEEE International Conference on Human-Robot Interaction (HRI '21 Companion), March 8-11, 2021, Boulder, CO, USA. ACM, New York, NY, USA, 4 pages. https://doi.org/ $10.1145 / 3434074.3447164$

\section{INTRODUCTION}

Mutual contingency, in which partners influence one another over the course of an exchange, is a governing principle of interaction [10]. Detecting contingency is a problem of determining social causality, or how the actions of one party are influenced by the preceding actions of other parties. Detecting contingency is critical for facilitating human-robot interaction because it enables robots to understand when they are expected to respond to human actions and when they are influencing people around them. In addition, contingency itself is a medium for planning and communication if a robot's influence on a person is known it can take actions that guide the interaction to a specific outcome.

In this work, we present a formalization for multi-agent contingency detection over discrete intervals of human behavior, implement an online interactive environment with multiple agents for remote data collection, and perform a preliminary data analysis to explore contingency detection in this environment. Participants play with two virtual agents and the resultant time-series data streams are segmented, correlated, and used to train a Support Vector Machine (SVMs) to determine which agent the participant is focused on. We find that our choice of cross-correlation features is not useful in training an SVM to determine contingency in a

Permission to make digital or hard copies of all or part of this work for personal or classroom use is granted without fee provided that copies are not made or distributed for profit or commercial advantage and that copies bear this notice and the full citation on the first page. Copyrights for components of this work owned by others than ACM must be honored. Abstracting with credit is permitted. To copy otherwise, or republish, to post on servers or to redistribute to lists, requires prior specific permission and/or a fee. Request permissions from permissions@acm.org.

HRI '21 Companion, March 8-11, 2021, Boulder, CO, USA

(C) 2021 Association for Computing Machinery.

ACM ISBN 978-1-4503-8290-8/21/03 . \$ \$15.00

https://doi.org/10.1145/3434074.3447164

\author{
Elaine Schaertl Short \\ Tufts University \\ Elaine.Short@tufts.edu
}

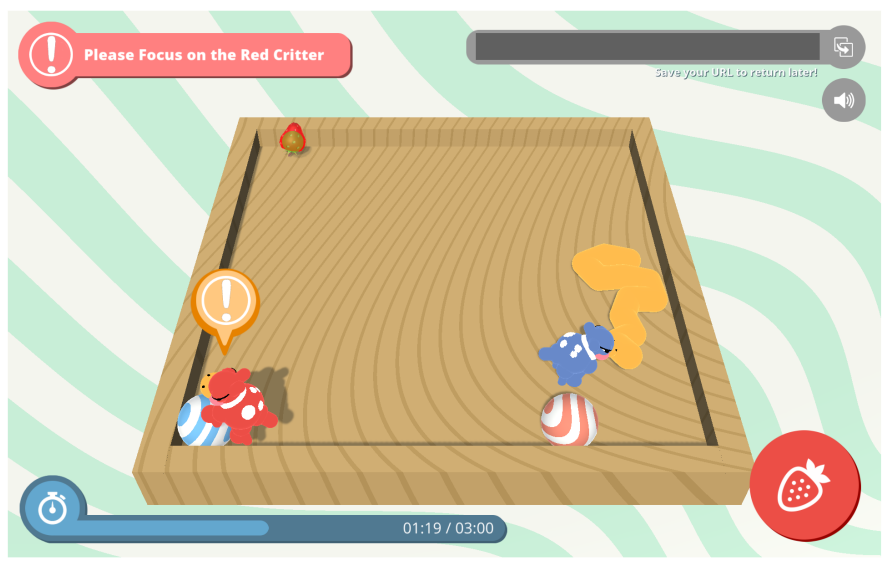

Figure 1: Experimental environment with two agents. The participant is instructed to play with one agent or another.

multi-agent environment, and discuss future directions towards an effective approach. The primary contribution of this work is an improved understanding of the difficulties of determining contingency between a human and multiple agents, as well as an interactive environment for collecting human-agent interaction data.

\section{RELATED WORK}

Contingency is a well-studied topic in developmental psychology. Adult voice and touch actions affect the responses of children with healthy expressive communication mechanisms, and the lack of contingent responses can be indicative of developmental issues [3]. Enabling a child to have contingent interactions during school can aid their learning [9] as well as their social and linguistic development. Similarly, contingent interaction can bootstrap more complex forms of play [5]. Participating in contingent interaction fosters the healthy development of the human mind.

Embodied agents that act with contingency can more effectively achieve social goals. For example, in one study, a robot collected a third more money at a charity event through just eye contact and facial feature mirroring [12]. In another study, the gaze contingency of virtual tutors improved learning in students [6].

In order to participate fluidly in contingent interactions an agent must be able to detect contingent behavior. Earlier work identified contingency detection as a two part process composed of detecting responses and interpreting their timing $[4,8]$. Temporal pattern analysis has been attempted successfully for detecting contingent behavior in human-robot interaction. These systems sometimes define contingency in strict terms, as a change in agent behavior within a finite time window in direct response to a signal from another agent, or in more general terms, as a correlation between robot behavior and changes in that robot's sensory environment $[2,7,10]$. 
Alternate approaches use information theory to hold a hypothesis about whether contingency is occurring and elicits signals to improve this hypothesis. This form of contingency detection is also called causal inference[11]. All of these approaches rely on hand-crafted features and context sensitive heuristics. In this paper we attempt to detect contingency from the first order derivatives of continuously generated signals produced by the participant and agents using a standard linear classifier.

\section{PROBLEM FORMULATION}

In this section we formalize the classification of a finite interval of interaction data as contingent or not. A person produces a multidimensional signal $\mathbf{h}(t)$ and an agent produces a multidimensional signal $\mathbf{r}(t)$. Each dimension of these signals describes a feature of behavior that changes over time. A classification over an interval has a label $l$ indicating which of the two agents has the users focus.

$$
l(t) \rightarrow\{0,1\}
$$

The task of this paper is to train a model given normalized training streams $\mathbf{h}(t)_{\text {train }}, \mathbf{r}(t)_{\text {train }}$ and a label $l$ for sample that predicts $l$ for normalized testing streams $\mathbf{h}(t)_{\text {test }}, \mathbf{r}(t)_{\text {test }}$.

\subsection{Interval Classification}

We assume we're given a set of non-overlapping intervals of human behavior $I$ from $\mathbf{h}(t)$. Each interval $I$ has a label $l$ over the entire interval.

$$
\begin{gathered}
I \equiv\left\{i_{1 \ldots n}=\left[t_{\left.i \_ \text {start } \ldots . . . t_{i \_ \text {end }}\right]}\right]\right. \\
l: I \rightarrow\{0,1\}
\end{gathered}
$$

In addition we use a window size of $\epsilon$ which defines how much time outside the interval of human behavior we can look at the agent behavior. Note that we do not need to separately define intervals since they can be defined in the terms above as $\left[t_{i}-\epsilon \ldots t_{j}+\epsilon\right]$. This window is necessary because the influential or influenced agent behavior is expected to lead or lag the participant's behavior, and so agent behavior which falls outside the interval of human behavior is like relevant.

The task is to train a model from a training subset $I_{\text {train }}$ that predicts $l$ over a testing subset $I_{\text {test }}$. Classification in this case is the equivalent of mapping from an interval to a label.

\subsection{Interval Classification}

We split our data into intervals with one consistent $l$. Note that the training label $l(t)$ indicates which agent the experimental interface is directing the participant towards, this agent is focused, and is not a ground truth label of which agent the participant is interacting with. It is expected that there is a lag between when $l$ switches agents, and when the participant redirects their attention.

We have three multidimensional streams of data for each interval: $\mathbf{h}(\mathrm{t})$ of the participant referenced above, $\mathbf{f}(\mathrm{t})$ of the focused agent and $\mathbf{u}(\mathrm{t})$ unfocused agent. In practice, the forward and backward looking windows over on either side of $t_{i \_s t a r t}$ and $t_{i \text { end }}$ may be different lengths to keep the intervals independent in cases where the participant intervals encroach on one another. i.e. where $t_{\text {i_end }}+\epsilon>t_{i+1 \_s t a r t}$.

3.2.1 Cross-Correlation. We compare the cross correlations between $\mathbf{h}(t)$ and both $\mathbf{f}(t)$ and $\mathbf{u}$. We compute the Pearson correlation coefficient $r$ pairwise between $n$ signals in $\mathbf{h}(t)$ and the $m$ signals in $\mathbf{f}(t)$ and $\mathbf{u}(t)$ with the agent signal shifted by a range $\{-j \ldots 0\}$ where $j$ is the number of samples shifted. This gives us a correlation value for each $j$ that describes how closely the two signals are correlated for each offset (where $r=1$ is perfect correlation, $r=-1$ is perfect inverse correlation and $r=0$ is no correlation). Equation 4 shows the $r$ for a given shift $j$ of signal $y(t)$ against signal $x(t)$

$$
r^{j}=\frac{\sum_{t=0}\left(x_{t}-\bar{x}\right)\left(y_{t+j}-\bar{y}\right)}{\sqrt{\sum_{t=0}\left(x_{t}-\bar{x}\right)^{2} \sum_{t=0}\left(y_{t+j}-\bar{y}\right)^{2}}}
$$

In this case $j \leq 0$ to limit the problem to detecting only the contingency between the agent and the participant, i.e. where the participants behavior is influenced by earlier agent behavior ${ }^{1}$. We then find the distribution of $r$ over values of $j$ and look for informative signals, which we define as having peaks of low variance within and between participants. We can train a simple model with this distribution data to perform a binary classification over intervals.

\section{METHODOLOGY}

We built and hosted an online platform to collect human-agent interaction data in pandemic conditions. The participant is presented with a virtual scene, show in figure 1, containing two animal-like agents. Initially the agents are asleep (inactive) and the participant is encouraged to familiarize themselves with the objects in the scene: balls, rope, and strawberries. After a short time the agents wake up and follow the behavior regime described in 4.2. The participant is then instructed to focus on one specific agent via onscreen prompts as well as a large exclamation point which hovers above the focused agent. Every 30 seconds, the focused agent switches. An onscreen timer shows how long the experiment has been running. After ten minutes the participant is thanked and dismissed, but is allowed to continue interacting as long as they desire.

The system, which we call HRI-Sandbox, is built to be configurable and generally useful for active learning. The version used for this paper implements behavioral-based robotics algorithms for the virtual agents, and uses the back-end for hosting and data storage only.

\subsection{Environment}

Our online platform serves a Unity WebGL application to humansubjects' browsers when they visit our study URL. We wrote a flask back-end that hosted a website to both serve the WebGL application and collect data from the interactions.

The participant can freely move items around the virtual scene. At regular intervals the data describing the participant's actions and the state of the environment is sent to the server. This includes the objects they touched, the position in the scene they clicked, the speed with which they dragged the mouse, the amount of time they

\footnotetext{
${ }^{1}$ The agent's behavior is designed to be contingent on the participant, so the relationship for $j>0$ is uninteresting
} 


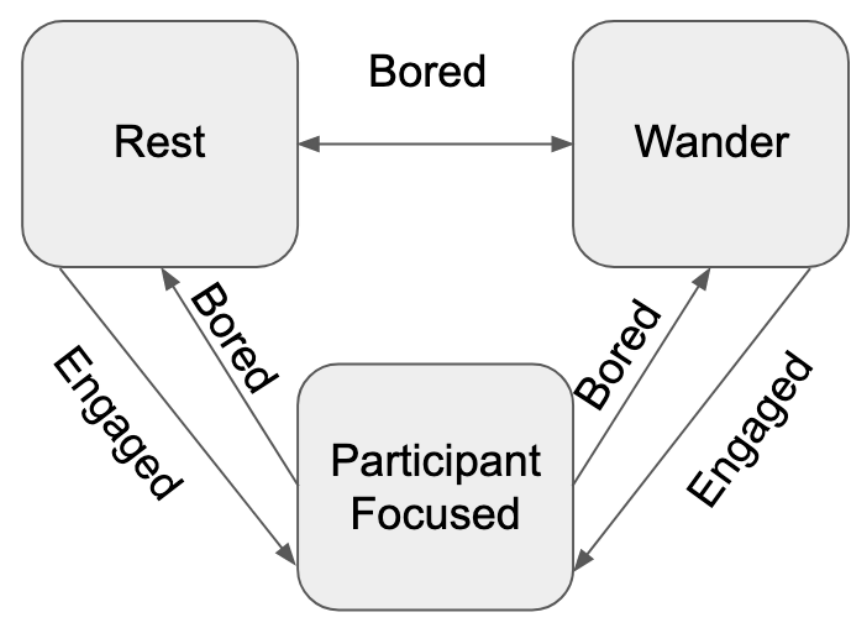

Figure 2: Finite State Machine describing agents behavior. Agents will oscillate between Rest and Wander based on their current focus and a timeout. They will focus on the participant if engaged $50 \%$ of the time.

held the mouse button down, which agent they were instructed to interact with, and any relevant information about the state of the agents. For this study we collected data at a rate of $5 \mathrm{~Hz}$.

When a participant visits the site they are given a unique user identifier (UUID). The UUID of the session is used to track the data associated with the interaction. In the future, we hope to allow participants to engage in longer term studies using this identifier.

\subsection{Agent Behavior}

The agents were programmed with the principles of Behavior Based Robotics in mind [1]. The agents were given the set of behaviors listed below.

(1) Look-Towards

(2) Move-Towards

(3) Grip / Grab

(4) Move-Away / Back-Up

(5) Bark / Emote

Look-Towards - The agent will look towards participant actions in its field of view.

Move-Towards - The agent will move towards a toy, food or any user action, unless its otherwise engaged.

Grip / Grab - The agent will grab a toy or eat food if the object is close to the agent's mouth for long enough (half a second).

Move-Away / Back-Up - The agent will back-away if a participant or agent is gripping the same toy as it. This is easily interpreted as tugging.

Bark / Emote - The agent will soundlessly bark or emote if it's being poked or led around in the absence of toys. It will also do this behavior at random times to proactively initiate interaction.

If the agent has to choose between these behaviors, it will focus on the participant half the time. These lower level behaviors and rules create opportunities for contingent reactions. For example throwing the ball in front of an agent will result in the agent playing fetch with the participant. Dragging the rope in front of an agent will result in tug-of-war.

In addition the agents obey a three-state Finite State Machine (FSM). The agents periodically switch between a Rest state where they look into the camera from a fixed position and emote, and a Wander state where they approach random objects in the scene (figure 2). The agents enter into a Participant Focused state when the participant seems to be interacting with them (e.g. waving a ball in front of an agent, tapping an agent, dragging a rope in front of the agent).

The agents were designed to show intuitive contingent behaviors when a participant interacted with them. Intuitive, in this case, is based on the expectations we think a reasonable person would have when playing with a virtual dog. For example dragging a rope to the agent results in the agent biting the rope. Shaking the rope lightly initiates a tug-of-war with the agent.

\section{EXPERIMENTAL RESULTS}

\subsection{Analysis and Discussion}

We extracted low-level features from the user's mouse movements and from the agent's position and orientation values. We did not consider the meta-state e.g. where the agent was in its FSM or what it was doing, because we were only interested in what was visually expressed to the user.

Analysis of cross-correlation based on correlations between the first and second order derivatives of participant and agent spatial behavior did not prove useful for classification using an SVM. There was no pattern in cross-correlation across all 24 participants as indicated by the lack of peaks and relatively large error bars in figure 3-left. The results improve when we look at individual participants (figure 3-right). The example participant shows a peak indicating the participants behavior is correlated with the focused agent's behavior two seconds in the past. These examples might indicate that the timing of a participants response is not generalizeable across all people and a more personal model is required for better classification.

We found that our SVM classifier did no better than random at determining contingency. There are several reasons why this might have been the case: First, it could have been due to the strong correlation between the two agent's behavior. We observed that the behavior of the two agents was highly correlated due to their following the same behavioral paradigm. While we don't have definitive proof, this high degree of correlation could affect multi-agent contingency detection if the signals used to determine participant focus on were too similar to usefully distinguish between the agents. Second, the features we chose might not show contingent relationships. We expected spatial behavior cross-correlation to show contingency because much of the interaction our environment enables involves tossing or dragging toys, but contingency in this environment might be event based rather than measurable from a continuous signal. Finally, since our study has no oversight, there's no guarantee that a participant is focusing on the agent they've been directed to focus on. Future iterations of this work will allow us to playback a session and confirm that participants are properly following the study instructions. 

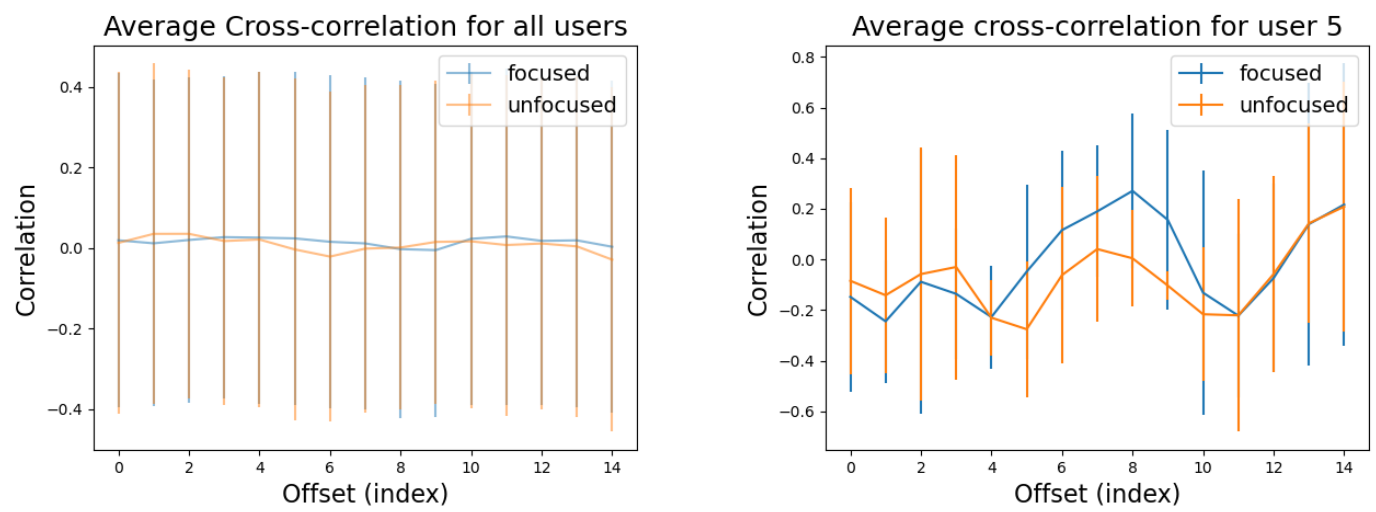

Figure 3: Cross correlation (y) between the participant and shifted agent velocity averaged across all participants (left) and for a single representative participant (right). The $x$-axis is the number of samples the agent signal was shifted by to produce the cross-correlation value ( 5 indices $=1$ second). Error bar is one standard deviation.

\section{FUTURE WORK}

We plan to continue building on our environment and agent behavior to determine what enables contingency detection between multiple agents. In this work, both agents followed the same behavioral regime without regard to the behavior of the other agent and we tried to detect contingency passively from the generated signals. In future work we will actively determine contingency by allowing the agents to communicate and produce more separable signals when the focus of the participant is more ambiguous. Greater ambiguity in the participant's behavior should result in more concerted efforts on the agents part to determine the participant's focus.

In addition, we only looked at visually apparent, continuous signals like velocity and rotational velocity. In the future we will incorporate discrete agent actions, like emotes and dances, into our model. The unstructured nature of our task (play with the agents) may have also contributed to the noise in the interaction, so in future work we will include at least one condition with turn-taking interactions and a more structured task. Incorporating discrete actions may also improve the estimates of deep learning approaches such as an LSTM network, which we plan to explore as an alternate classifier.

\section{REFERENCES}

[1] Rodney A Brooks. Intelligence without representation. Cambridge, MA: MIT Press, 1997.

[2] Vivian Chu, Kalesha Bullard, and Andrea L Thomaz. Multimodal real-time contingency detection for hri. In 2014 IEEE/RSf International Conference on
Intelligent Robots and Systems, pages 3327-3332. IEEE, 2014.

[3] Cynthia J. Cress, Jodi Grabast, and Kimberly Burgers Jerke. Contingent Interactions Between Parents and Young Children With Severe Expressive Communication Impairments. Communication Disorders Quarterly, 34(2):81-96, 2013. eprint: https://doi.org/10.1177/1525740111416644

[4] Kevin Gold and Brian Scassellati. Learning acceptable windows of contingency. Connection Science, 18(2):217-228, 2006.

[5] Tiffany L Jimerson and Lynne A Bond. Mothers' epistemologies, turn-taking, and contingent interaction with preschoolers. Fournal of Applied Developmental Psychology, 22(4):379-396, 2001. Publisher: Elsevier.

[6] Hanju Lee, Yasuhiro Kanakogi, and Kazuo Hiraki. Social Appearance of Virtual Agent and Temporal Contingency Effect. In Proceedings of the 3rd International Conference on Human-Agent Interaction, HAI '15, pages 85-89, New York, NY, USA, 2015. Association for Computing Machinery. event-place: Daegu, Kyungpook, Republic of Korea.

[7] J. Lee, J. F. Kiser, A. F. Bobick, and A. L. Thomaz. Vision-based contingency detection. In 2011 6th ACM/IEEE International Conference on Human-Robot Interaction (HRI), pages 297-304, 2011.

[8] Javier R Movellan. An infomax controller for real time detection of social contingency. In Proceedings. The 4th International Conference on Development and Learning, 2005, pages 19-24. IEEE, 2005.

[9] Sarah Roseberry, Kathy Hirsh-Pasek, and Roberta M Golinkoff. Skype me! Socially contingent interactions help toddlers learn language. Child development, 85(3):956-970, 2014.

[10] Elaine Schaertl Short, Mai Lee Chang, and Andrea Thomaz. Detecting contingency for hri in open-world environments. In Proceedings of the 2018 ACM/IEEE International Conference on Human-Robot Interaction, HRI '18, page 425-433, New York, NY, USA, 2018. Association for Computing Machinery.

[11] George M Tarabulsy, Rejean Tessier, and Arvid Kappas. Contingency detection and the contingent organization of behavior in interactions: Implications for socioemotional development in infancy. Psychological Bulletin, 120(1):25, 1996.

[12] P. Wills, P. Baxter, J. Kennedy, E. Senft, and T. Belpaeme. Socially contingent humanoid robot head behaviour results in increased charity donations. In 2016 11th ACM/IEEE International Conference on Human-Robot Interaction (HRI), pages 533-534, 2016. 